\title{
Distribución de beta-catenina en lesiones displásicas de colon en ratas alimentadas con leche de búfala
}

\author{
Catuogno, M.S.; Montenegro, M.A.; S.Negrette, M. \\ Cátedra de Patología General y Sistemática, Facultad de Ciencias Veterinarias, UNNE. \\ Sargento Cabral 2139, Corrientes 3400, Argentina. E-mail: patgral@vet.unne.edu.ar
}

\begin{abstract}
Resumen
Catuogno, M.S.; Montenegro, M.A.; S.Negrette, M.: Distribución de beta-catenina en lesiones displásicas de colon en ratas alimentadas con leche de búfala. Rev. vet. 29: 2, 8387, 2018. El objetivo del presente trabajo fue determinar la distribución de beta-catenina en lesiones displásicas (LD) de colon en ratas alimentadas con leche de búfala. Se utilizaron ratas Wistar machos de 6 semanas, que recibieron diferentes dietas lácteas durante 123 y 240 días. Se suministraron dos tipos de dietas lácteas, una estándar, proveniente de búfalas alimentadas con pasturas naturales, y otra mejorada obtenida de búfalas suplementadas con aceite de pescado, con alto contenido en ácido linoleico conjugado y omega-3. Las LD fueron inducidas por el carcinógeno 1,2-dimetil-hidrazina. Para detectar beta-catenina se empleó la técnica de inmunohistoquímica. Las células epiteliales colónicas mostraron claramente expresión membranosa de beta-catenina. En células superficiales, la proteína pudo ser identificada también en el citoplasma, pero con una tinción membranosa más débil. En las células que estaban localizadas hacia la profundidad de la mucosa, se observó falta de proteína en el citoplasma, permaneciendo en la membrana citoplasmática. Los enterocitos ubicados sobre la muscular de la mucosa mostraron tinción membranosa débil en comparación con las membranas de las células superficiales. No se observó beta-catenina en núcleos de células normales. En LD de ratas alimentadas con leche de búfala, la distribución de beta-catenina cambió sustancialmente acorde a la severidad de la displasia. Estos hallazgos podrían ser de utilidad para la detección de lesiones pre-carcinomatosas en estadios tempranos.
\end{abstract}

Palabras clave: ratas, leche de búfala, lesiones displásicas, beta catenina.

\begin{abstract}
Catuogno, M.S.; Montenegro, M.A.; S.Negrette, M.: Distribution of beta-catenin in colon dyplastic lesions of rats fed with buffalo milk. Rev. vet. 29: 2, 83-87, 2018. The aim of this study was to determine the distribution of beta-catenin in cells of dysplastic lesions (DL) in rats fed with buffalo milk. Six-week Wistar male rats were administered with different milk diets during 123 and 240 days. Animals were fed with two diets: milk from buffaloes fed on natural pastures, and milk from buffaloes supplemented with fish oil containing high concentration of conjugated linoleic acid and omega-3. DL were induced by 1,2-dimethylhydrazine. Immunohistochemistry was performed to detect beta-catenin. The colonic epithelial cells clearly displayed beta-catenin membranous expression. In superficial cells, the protein could also be identified in cytoplasm with a weaker stain. In cells located deep in the mucosa it was observed a lack of protein in the cytoplasm, remaining only in the cytoplasmic membrane, thus being cytoplasm negative to the presence of beta-catenin. The enterocytes located on the muscular layer of mucosa showed a weaker membranous stain compared to superficial cell membranes. Beta-catenin in the nucleus of normal cells was not observed. In DL of buffalo milk fed rats the beta-catenin distribution substantially changed according to severity of dysplastic lesions. These findings could be useful in the detection of pre-carcinomatous lesions at early stage.
\end{abstract}

Key words: rats, buffalo milk, dysplastic lesions, beta catenin.

\section{INTRODUCCIÓN}

Mediante estudios clínicos se ha demostrado una reducción de la hiperproliferación de la mucosa intes-

Recibido: 14 febrero 2018 / Aceptado: 6 abril 2018 tinal después del consumo de ácidos grasos poliinsaturados omega-3 derivados del aceite de pescado, en individuos con elevado riesgo de cáncer de colon debido a la presencia de adenomas colónicos esporádicos ${ }^{1,2}$.

Aunque algunos investigadores describieron mecanismos a través de los cuales los ácidos grasos omega-3 
tienen una participación en la carcinogénesis actuando en la biosíntesis de eicosanoides en la peroxidación lipídica y en alguna vía de transducción de señal, en la actualidad aún son escasos los datos que relacionen todos estos mecanismos ${ }^{13}$.

En referencia al ácido linoleico conjugado (ALC), se le han atribuido numerosas propiedades biológicas como agente anticarcinogénico, antiarteroesclerótico, antiadipogénico, y antidiabetogénico. Además, el ALC modula la inmunidad y los mecanismos de trombosis, así como la bioquímica de los ácidos grasos, el metabolismo lipídico y la expresión genética en el hígado, músculo y tejido adiposo ${ }^{3}$.

La concentración de ALC en la leche bovina es variable, alternando desde 2,4 a 21,8 $\mathrm{mg}$ de ALC/g de grasa ${ }^{24}$. Por otra parte, un estudio demostró que en la leche de los rumiantes su concentración podría aumentar por la adición dietaria de aceite de girasol (rico en ácido linoleico) y aceite de lino (alto en ácido linolénico) ${ }^{9}$.

Como otros ácidos grasos poliinsaturados, el ALC puede modular la carcinogénesis mediante mecanismos que afectan los estadios del cáncer conocidos como iniciación, promoción, progresión y/o regresión ${ }^{7}$.

Un biomarcador intermedio utilizado para detectar cáncer de colon son los focos de criptas aberrantes (FCA), lesiones ampliamente usadas para detectar sustancias promotoras y supresoras. Se postula que los FCA son lesiones pre-malignas. La displasia se define como un "cambio pre-carcinomatoso" y como "una alteración neoplásica inequívoca del epitelio colónico" ${ }^{23}$. También, se han incluido a las criptas que acumulan $\beta$-catenina como nuevos biomarcadores de la carcinogénesis colónica en ratas, por su fuerte predisposición al cáncer de colon ${ }^{10,16}$.

Las cateninas son elementos necesarios para el establecimiento de las adhesiones intercelulares, constituyendo factores importantes para la regulación de los complejos de adhesión. Una de ellas, la catenina beta, actúa como intermediaria para la unión entre las moléculas de adhesión y el citoesqueleto celular. En los mamíferos se localiza en el citoplasma y núcleo de la célula, participando en la formación de uniones adherentes de epitelios, siendo además un componente importante en la regulación transcripcional, en particular durante el desarrollo del embrión ${ }^{28,30}$.

Existen mutaciones que inactivan la $\beta$-catenina y/o el gen APC (adenomatous polyposis coli); este último es un supresor tumoral que codifica una proteína inhibidora del cáncer colorrectal. Por su parte, las vías de señalización WNT (wingless type) son un grupo de proteínas transductoras que transfieren señales del exterior al interior de una célula. La estimulación de la vía WNT conduce a la acumulación de $\beta$-catenina ${ }^{10,16}$.

La pérdida de la función APC imposibilita la disminución de $\beta$-catenina, la cual consecuentemente se acumula en el citoplasma y se traslada al núcleo donde se complementa con factores de transcripción y activa genes que representan una vía oncogénica potencial ${ }^{10,12,16}$.
La expresión aberrante de $\beta$-catenina en tumores de colon y el descubrimiento de mutaciones de $\beta$-catenina en pequeños adenomas, sugieren que tales alteraciones son eventos tempranos en la carcinogénesis colorrectal en seres humanos. Las células epiteliales colónicas normales adyacentes a los FCA muestran fuerte expresión membranosa de $\beta$-catenina y falta de expresión citoplasmática y nuclear. En FCA displásicos, la expresión membranosa reducida de $\beta$-catenina estuvo asociada al aumento de la expresión citoplasmática y nuclear. La expresión membranosa de $\beta$-catenina fue reducida y la expresión citoplasmática y nuclear aumentada en FCA acorde a sus grados de displasia. Estos datos sugieren que los FCA y su expresión aberrante de $\beta$-catenina participan en la tumorigénesis colónica ${ }^{8}$.

Cuando los genes para $\beta$-catenina o APC están mutados o la vía de señalización WNT está activada, la $\beta$-catenina se acumula en el citosol, liga proteínas de la familia de factor de células $\mathrm{T}$ y de transcripción y se mueve al núcleo ${ }^{14}$. La activación de la vía $A P C / \beta$ catenina participa de manera importante en la tumorigenesis colónica y las mutaciones de APC ${ }^{18}$ y $\beta$-catenina ${ }^{27}$ ocurren de manera temprana en este proceso.

Estos datos sugieren que, además de la estabilidad proteica alterada (que ocurre cuando las serinas $33 \mathrm{y} / \mathrm{o}$ 45 se encuentran mutadas), la exposición al carcinógeno también resulta en aumento de transcripción o estabilización de $\beta$-catenina. Por otra parte, mutaciones de $\beta$-catenina se han encontrado en el hígado ${ }^{5,17}$, colon ${ }^{29}$, tumores de vejiga ${ }^{25}$ y en criptas colónicas aberrantes de ratones ${ }^{30}$ y tumores de ratas ${ }^{11,28}$.

La presencia de $\beta$-catenina en LD de colon de ratas podría coadyuvar en la detección temprana de lesiones pre-carcinomatosas.

\section{MATERIAL Y MÉTODOS}

Para la experiencia se utilizaron ratas Wistar machos de 6 semanas $(160 \mathrm{~g})$ que recibieron diferentes dietas lácteas durante 123 y 240 días ${ }^{21}$. Se utilizaron dos tipos de dietas lácteas, una estándar, obtenida de búfalas alimentadas con pasturas naturales, y otra dieta láctea mejorada proveniente de búfalas suplementadas con aceite de pescado, con alto contenido en ALC y Omega-3. Se conformaron tres lotes experimentales de la siguiente manera:

Lote 1: ratas con agua y alimento balanceado comercial ad-libitum durante 123 y 240 días + inyecciones de 1,2-dimetilhidrazina (DMH).

Lote 2: ratas con alimento balanceado comercial y leche ad-libitum de búfalas alimentadas con pasturas naturales durante 123 y 240 días + inyecciones con DMH.

Lote 3: ratas con dieta y leche ad-libitum de búfalas suplementadas con maíz y aceite de pescado, durante 123 y 240 días + inoculación con DMH.

La inducción de lesiones displásicas se realizó mediante inyecciones subcutáneas de DMH a la dosis de $20 \mathrm{mg} / \mathrm{kg}$, a partir de los 15 días del suministro de la 
dieta láctea, y se prolongó durante 35 días, totalizando 5 inyecciones, una por semana. Luego del periodo de inyección, las ratas continuaron alimentándose con leche, hasta completar 123 y 240 días de experiencia, luego de los cuales se realizó el sacrificio.

Posteriormente al sacrificio, el intestino grueso se fijó en formol bufferado al $10 \%$ para luego ser procesados según la técnica clásica para bloques parafinados, cortados en $5 \mu \mathrm{m}$ y coloreados con hematoxilina-eosi$n a$. En tales cortes coloreados, las lesiones displásicas fueron clasificadas de acuerdo al grado de severidad de la displasia en leves, moderadas y severas ${ }^{26}$.

Más adelante se procedió a efectuar cuatro cortes seriados de las lesiones displásica observadas con dichos colorantes. Los cortes se colocaron en portaobjetos previamente tratados pon poly-l-lisyne a fin de evitar que se despeguen durante el procesamiento.

Con cada una de las muestras obtenidas se procedió de la siguiente manera: desparafinado e hidratación; recuperación antigénica en microondas con las muestras sumergidas en buffer citrato $\mathrm{pH}$ 6; inactivación de la peroxidasa endógena con metanol y peróxido de hidrógeno; bloqueo de uniones inespecíficas con suero normal de cabra diluido $1 / 20$ con phosphate buffered saline (PBS) a pH 7,4; incubación con "anticuerpo primero" (anti-beta catenina: Purified Mouse Anti-Beta Catenin Transduction Laboratories, utilizado en dilución $1 / 200$ ) en cámara húmeda a $4^{\circ} \mathrm{C}$, durante toda la noche.

Luego se realizó incubación con "anticuerpo segundo" (biotinilado policlonal anti- IgG de ratón conjugado con biotina, código AM-B1). Este anticuerpo es elaborado en el Laboratorio de Endocrinología y Tumores Hormonodependientes de la Facultad de Bioquímica y Ciencias Biológicas de la Universidad Nacional del Litoral (Argentina).

La dilución óptima de uso fue 1/100. La incubación se realizó en cámara húmeda a temperatura de $25^{\circ} \mathrm{C}$ durante 30 minutos, y el revelado se hizo con ExtrAvidin ${ }^{\circledR}$ (peroxidase buffered aqueous solution, SigmaAldrich) diluido 1/200 con PBS estéril. Se dejó 30 minutos en cámara húmeda a $25^{\circ} \mathrm{C}$.

El cromógeno (Sigma-Aldrich) se utilizó en una dilución final de $0,05 \%$ con diaminobenzidina tetrahidrocloruro $+0,015 \%$ de peróxido de hidrógeno en PBS $0,01 \mathrm{M} \mathrm{pH} \mathrm{7,2.} \mathrm{Se}$ incubaron las muestras durante $2 \frac{1}{2}$ minutos a temperatura ambiente, luego se efectuó la deshidratación de los cortes y montaje con bálsamo de Canadá (Biopack). Una vez obtenidas las muestras coloreadas con inmunohistoquímica (IHQ) se procedió a su análisis microscópico evaluando:

a) diferencias en la cantidad de proteína $\beta$-catenina de las lesiones en comparación con las criptas normales circundantes (a mayor coloración, mayor cantidad de proteína). b) distribución de las proteínas en citoplasma o núcleo. Normalmente las proteínas se localizan en la membrana citoplasmática.

c) posibilidad de poder determinar diferencias entre focos displásicos leves, moderados o severos con inmunohistoquímica de $\beta$-catenina.

d) diferencias entre focos displásicos dependiendo del tipo de leche ingerida por los animales.

Todos los análisis efectuados se llevaron a cabo teniendo en cuenta como control interno la mucosa normal circundante a las lesiones displásicas.

\section{RESULTADOS}

Las células epiteliales colónicas de la mucosa intestinal histológicamente normal, claramente mostraron predominio de expresión membranosa de $\beta$-catenina. En las células superficiales más diferenciadas se pudo identificar a la proteína también en el citoplasma, con coloración mucho más leve que en la membrana celular.

No sucedió lo mismo en las células de la profundidad de las glándulas, en las que el citoplasma resultó negativo para la presencia de $\beta$-catenina, quedando solamente en la membrana citoplasmática. Los enterocitos próximos a la capa muscular de la mucosa presentaron coloración membranosa mucho mas clara que las células superficiales. No se observó $\beta$-catenina en los núcleos de células normales.

En las lesiones displásicas la distribución de $\beta$-catenina se modificó sustancialmente acorde a la severidad de la displasia. En las displasias leves (Figura 1), se observó distribución de $\beta$-catenina en membrana semejante a las glándulas normales, pero con notable acúmulo en citoplasma, cuando las displasias afectaban a la profundidad de las criptas.

En las lesiones displásicas de grado moderado (Figura 2), con inmunohistoquímica se observó claramente mayor acúmulo de proteína en las glándulas displásicas al compararlas con las glándulas normales circundantes. La marcación de $\beta$-catenina fue intensa en las membranas de células displásicas, que además presentaron acumulación de $\beta$-catenina en los núcleos.

Las lesiones displásicas inducidas y encontradas en todos los animales experimentales de este estudio fue-

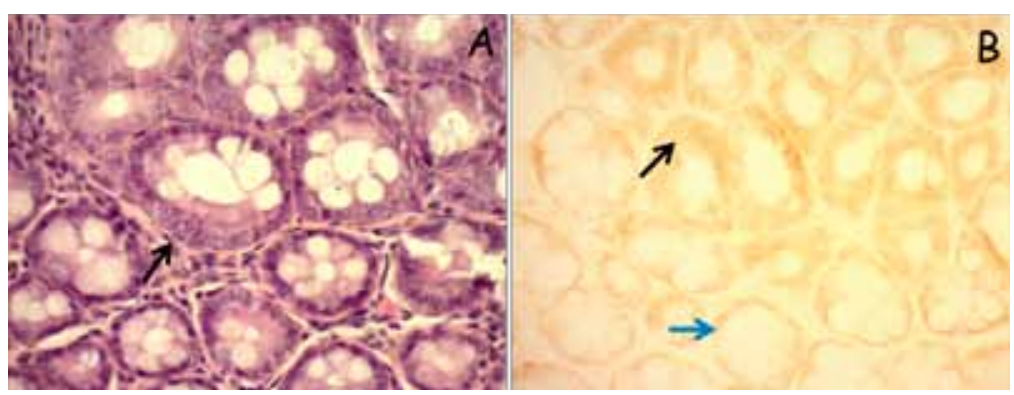

Figura 1. A: displasia leve (flecha). HE 40X. B: el mismo foco de criptas con marcación de beta-catenina, expresada principalmente en la membrana citoplasmática al igual que lo observado en células normales (flecha superior), y acumulación en citoplasma de células displásicas (flecha inferior). IHQ 40X. 

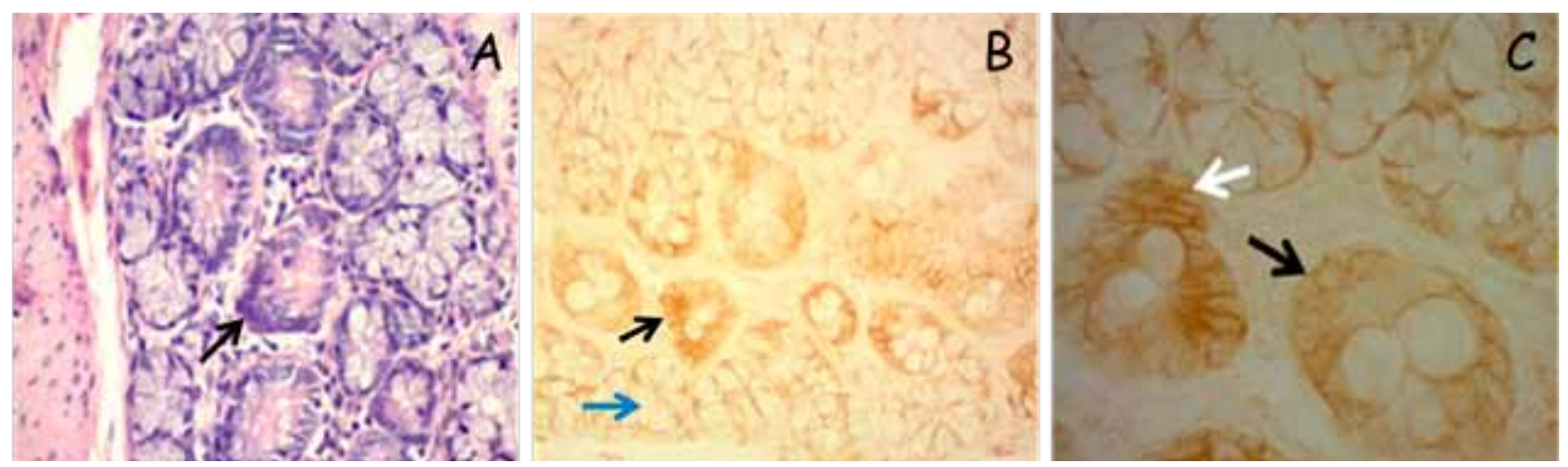

Figura 2. A: displasia moderada (flecha). HE 40X. B: en el mismo foco de criptas las células displásicas muestran mayor expresión de beta-catenina (flecha superior) comparada con las células normales (flecha inferior). IHQ 40X. C: las células displásicas muestran tinción membranosa más fuerte (flecha blanca) y acúmulo de betacatenina intracelular (flecha negra). IHQ 1000X.

ron clasificadas como leves y moderadas, no habiéndose observado lesiones de tipo severas o carcinomas in situ, por lo que no se obtuvieron datos de distribución de $\beta$-catenina en este tipo de lesiones. Tampoco hubo diferencias en la distribución de $\beta$ - catenina entre los diferentes lotes experimentales.

\section{DISCUSIÓN}

En seres humanos, el descubrimiento de la expresión aberrante de $\beta$-catenina en una proporción significativa de focos de criptas displásicas, sugiere que la disfunción de la $\beta$-catenina es un evento muy temprano en el desarrollo de tumores colónicos. El hecho de que las alteraciones fenotípicas aumentaron significativamente a medida que el grado de displasia aumentaba, indica que cada una de estas alteraciones fenotípicas refleja el rol de la $\beta$-catenina en la progresión de las $\mathrm{LD}^{8}$.

Estos hallazgos son coincidentes con nuestra experiencia en la cual encontramos que las LD mostraron marcación de $\beta$-catenina diferente a las glándulas normales, y su distribución varió de acuerdo al grado de displasia. En células normales, bien diferenciadas, la $\beta$-catenina se localiza en la membrana para cumplir su función de adherencia a las células vecinas, mientras que en células menos diferenciadas, como ocurre en las LD, se reduce la expresión membranosa, asociándose con mayor expresión en citoplasma y posteriormente en el núcleo, acorde va aumentando la severidad de la displasia.

Investigadores han reportado un significativo aumento de la expresión nuclear y disminución de la expresión membranosa de $\beta$-catenina en carcinomas, comparados con sus adenomas adyacentes ${ }^{8}$. La expresión citoplasmática de $\beta$-catenina tambien ha sido reportada en lesiones displásicas de ratas tratadas con azoximetano ${ }^{19,20}$. Los mecanismos que llevan a la acumulación citoplasmática y a la expresión de $\beta$-catenina son inciertos.

También se encontró sobreexpresión de $\beta$-catenina en adenomas colónicos de ratas inducidas con azoximetano. La $\beta$-catenina también se localizó en el núcleo de adenomas de este mismo modelo, en el cual la ma- yoría de los tumores había demostrado tener APC intacto ${ }^{4}$ sugiriendo que APC no es un objetivo común del azoximetano.

Según algunos autores la carcinogénesis experimental colónica en ratas puede ser inhibida alimentándolas con dietas con altas concentraciones de aceite de pescado ${ }^{6,22}$ o suplementadas con ácidos grasos omega-3 ${ }^{15}$. Por otra parte, ALC puede modular la carcinogénesis mediante mecanismos que afectan los estadios del cáncer conocidos como iniciación, promoción, progresión y/o regresión. Sin embargo, el mecanismo actual por el cual el ALC afecta la carcinogénesis todavía es incierto. Un estudio sugiere que el ALC podría actuar mediante mecanismos antioxidantes ${ }^{7}$.

En nuestra experiencia, hemos observado menor frecuencia de LD en ratas que recibieron leche mejora$\mathrm{da}^{22}$, sin embargo, la distribución de $\beta$-catenina en LD inducidas con DMH en ratas alimentadas con leche de búfala estándar y mejorada con mayor concentración de ALC y omega-3, fueron semejantes. En el presente trabajo, la distribución de $\beta$-catenina en LD de colon de ratas fue coincidente con las LD halladas en colon de seres humanos. Estos hallazgos podrían ayudar en la detección temprana de lesiones pre-carcinomatosas.

\section{REFERENCIAS}

1. Anti M, Marra G, Armelao F. 1992. Effects of omega-3 acids on rectal cell proliferation in subjects at risk for colon cancer. Gastroenterology 103: 883-891.

2. Anti M, Armelao F, Marra G. 1994. Effects of different doses of fish oil on rectal cell proliferation in patients with sporadic colonic adenomas. Gastroenterology 107: 17091718.

3. Belury MA. 2002. Dietary conjugated linoleic acid in health: physiological effects and mechanisms of action. Ann Rev Nutr 22: 505-531.

4. Caderni G et al. 1997. APC mutations in aberrant crypt foci (ACF) and colonic tumors induced by azoximethane in F 344 rats. Proc Amer Assoc Cancer Res 38: 467.

5. Calvisi DF, Factor BM, Loi R, Thorgeirsson SS. 2001. Activation of $\beta$-catenin during hepatocarcinogenesis in 
transgenic mouse models: Relationship to phenotype and tumor grade. Cancer Res 61: 2085-2091.

6. Deschner EE, Lytle JS, Wong G, Ruperto JF, Newmark HS. 1990. The effect of dietary omega-3 fatty acids (fish oil) on azoximethanol-induced focal areas of dysplasia and colon tumor incidence. Cancer 66: 2350-2356.

7. Ha YL, Storkson JM, Pariza MW. 1990. Inhibition of benzo(a)pyrene-induced mouse fore-stomach neoplasia by conjugated dienoic derivatives of linoleic acid. Cancer Res 50: 1097-1101.

8. Hao XP, Pretlow TG, Rao S, Pretlow TP. 2001. $\beta$-Catenin expression is altered in human colonic aberrant cripta foci. Cancer Res 61: 8085-8088.

9. Kelly ML et al. 1998. Dietary fatty acid sources affect conjugated linoleic acid concentrations in milk from lactating dairy cows. J Nutr 128: 881-885.

10. Kinzler KW, Vogelstein B. 1996. Lessons from hereditary colorectal cancer. Cell 87: 159-170.

11. Koesters $\mathbf{R}$ et al. 2001. Predominant mutation of codon 41 of the $\beta$-catenin proto-oncogene in rat colon tumors induced by 1,2-dimethylhydrazine using a complete carcinogenic protocol. Carcinogenesis 22: 1885-1890.

12. Lamlum H, Papadopoulos A, Llyas M. 2000. APC mutations are sufficient for the growth of early colorrectal adenomas. Proc Natl Acad Sci USA 95: 2225-2228.

13. Larsson S, Kumlin M, Ingleman M, Wolk A. 2004. Dietary long chain n-3 fatty acids for the prevention of cancer: a review. Am J Clin Nutr 79: 935-945.

14. Mann B et al. 1999. Target genes of $\beta$-catenin-T cell-factor/lymphoid-enhancer-factor signaling in human colorrectal carcinomas. Proc Natl Acad Sci USA 96: 1603-1608.

15. Minoura T et al. 1998. Effect of dietary eicosapentaenoic acid on azoximethane induced colon carcinogenesis in rats. Cancer Res 48: 4790-4794.

16. Møllersen L, Vikse R, Andreassen A. 2004. Adenomatous polyposis coli truncation mutations in 2-amino1-methyl-6-phenylimidazol 4,5b pyridine (PhIP)-induced intestinal tumours of multiple intestinal neoplasia mice. Mutat Res 557: 29-40.

17. Ogawa K, Yamada Y, Kishibe K, Ishizaki K, Tokusashi Y. 1999. $\beta$-catenin mutations are frecuent in hepatocellular carcinomas but absent in adenomas induced by diethylnitrosamine in B6C3F1 mice. Cancer Res 59:1830-1833.

18. Powel SM et al. 1992. APC mutations occur early during colorectal tumorigenesis. Nature 359: 235-237.
19. Pretlow TP, Bird RP. 2000 . Frequent $\beta$-catenin gene mutations and accumulations of the protein in the putative preneoplastic lesions lacking macroscopic aberrant crypt foci appearance, in rat colon carcinogenesis. Cancer Res 60: 3323-3327.

20. Pretlow TP, Bird RP. 2001. Sequential analysis of morphological and biological properties of $\beta$-cateninaccumulated crypts, provable premalignant lesions independent of aberrant crypt foci in rat colon carcinogenesis. Cancer Res 61: 1874-1878.

21. Ramírez GV et al. 2012. Disminución del desarrollo de cáncer experimental del colon en ratas alimentadas con leche de búfala. Rev Vet 23: 15-19.

22. Reddy BS, Marayama H. 1986. Effects of dietary fish oil on azoximethane induced colon carcinogenesis in male F344 rats. Cancer Res 46: 3367-3370.

23. Riddell RH et al. 1983. Dysplasia in inflammatory bowel disease: standardized classification with provisional clinical applications. Hum Pathol 14: 931-966.

24. Riel RR. 1963. Physico-chemical characteristics of canadian milk fat. Unsaturated milk fatty acids. J Dairy Sci 46 : 102-106.

25. Shiina $\mathbf{H}$ et al. 2001. Alterations of beta and gamma catenin in N-butyl-N-(-4-hydroxybutyl) nitrosamine-induced murine bladder cancer. Cancer Res 61: 7101-7109.

26. Siu IM, Pretlow TG, Amini SB, Pretlow TP. 1997. Identification of dysplasia in human colonic aberrant crypt foci. Am J Pathol 150: 1805-1813.

27. Sparks AB, Morin PJ, Vogelstein B. 1998. Mutational analysis of the APC/ $\beta$-catenin/Tcf pathway in colorrectal cancer. Cancer Res 58: 1130-1134.

28. Takahashi M, Fukuda K, Sugimura T, Wakabayashi K. 1998. $\beta$-catenin is frequently mutated and demonstrates altered cellular location in azoximethane induced rat colon tumors. Cancer Res 58: 42-46.

29. Takahashi M, Nakatsugi S, Sugimura T, Wakabayashi K. 2000. Frecuent mutations of beta-catenin in mouse colon tumors induces by azoxymethane. Carcinogenesis 21 : 1117-1120.

30. Yamada Y et al. 2001. Sequential analysis of morphological and biological properties of beta-catenin accumulated crypts, provable premalignant lesions independent of aberrant crypt foci in rat colon carcinogenesis. Cancer Res 61: 1874-1878. 